\section{Epigenetics: where environment, society and genetics meet}

\author{
"The goal is to use epigenetics to anticipate health in the individual and, \\ more importantly, the population. Before this can be done, several \\ challenges must be faced."
}

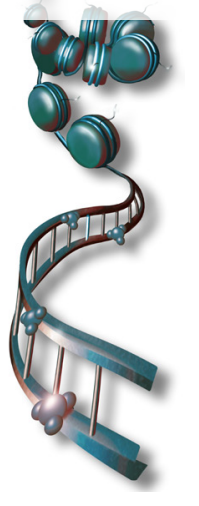

Keywords: environment $\bullet$ epigenetics $\bullet$ noncoding RNA • socioeconomic status - transgenerational epigenetic inheritance

Understanding how environment, social factors and genetics combine to affect patterns of health is an urgent priority. Social factors that affect health patterns include race and socioeconomic status (SES), while biological factors include sex, genetics and epigenetics. The consequence of biology and environment can be observed in life expectancy data. At 25 years old, life expectancy for white men is 4.4 years greater than for African-American men. Females follow a similar pattern. Income also affects life expectancy with high income earners outliving their low income counterparts, and white males and females outliving African-American males and females by 2-4 years at every income level [1]. Environment also affects perinatal mortality. US-born African-American women experience greater rates of perinatal mortality than their foreign-born counterparts [2]. Similar patterns can be observed with multiple health conditions, including cancer, depression, diabetes and heart disease highlighting the importance of this issue [1]. What is less clear is the mechanism by which these disparities are caused. One way in which biology and environment can both leave their fingerprint is through epigenetic changes.

Epigenetics refers to the regulation of gene expression through modifications to DNA or DNA-associated proteins. Epigenetic regulation includes histone modifications, DNA methylation and nonprotein coding RNA. DNA methylation has been a particular focus of environment-mediated changes in health. Methylation changes have been associated with environmental chemical exposure [3], race, gender [4] and income level [5]. However, epigenetic gene regulation is bigger than methylation alone.

Human and animal studies have begun to investigate environmental effects on epigenetics. While these publications are increasing, some factors need to be considered, these include tissue specificity, epigenetic specificity and transgenerational studies.

\section{Tissue specificity}

Tissue-specific epigenetic profiles are well accepted, and so it must be noted that blood cells also have cell-specific profiles. Circulating blood is a convenient source of material for epigenetic testing. It is minimally invasive to collect and can be collected multiple times over a period of time. However, development and environment changes the cellular makeup of blood.

Cellular heterogeneity may confound epigenetic results, since epigenetics differs from one blood cell to another [6,7]. For example, neutrophils predominate blood of a newborn, but, by 2-4 years, lymphocytes are the majority population [8]. Furthermore, cord blood also varies in its cellular make up compared to peripheral blood [9]. These two factors can confound the comparison of methylation profiles from a newborn to an adult, even in the same individual. The constituents of the blood can also be changed if the individual is experiencing inflammation, an infection or high amounts of stress [10-12]. All of these factors can be influenced by an individual's SES. These differences highlight

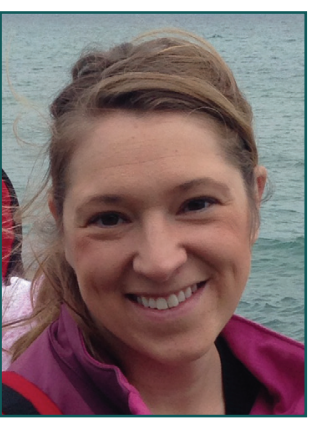

Amber V Majnik

Author for correspondence: Department of Pediatrics Medical College of Wisconsin, Milwaukee, WI, USA

Tel.: +1 4149552374

amajnik@mcw.edu

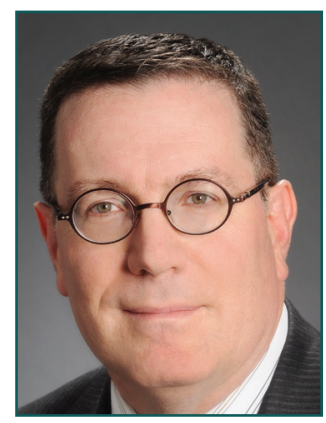

Robert H Lane

Department of Pediatrics Medical College of Wisconsin, Milwaukee, WI, USA 
how developmental and environmental changes can alter epigenetic results.

The importance of different epigenetic patterns between specific cell types and whole blood will vary depending on disease or condition. Subtle differences are likely more important for multifactorial diseases, like obesity, where the environment, genetics and social factors all contribute to pathology. Furthermore, while methylation patterns may appear similar between cell types, changes in histone code or noncoding RNA may differ.

\section{Epigenetic specificity}

We believe social and environmental factors rely on epigenetics to effect biologic change. The study of how the environment affects phenotype is environmental epigenetics. A cell has many epigenetic tools to modify its gene expression in response to the environment. However, to understand environmental epigenetics, we must look beyond gene expression and protein coding regions and pay attention to regulatory and noncoding regions. This includes studying methylation and histone modifications, as well as noncoding RNA.

The majority of SES studies give a limited view of the epigenetic landscape. The majority of these studies investigate SES and changes in global methylation. Some studies have taken an extra step to investigate methylation in disease relevant genes. However, to fully appreciate the epigenetic impact of the environment, analysis must go beyond methylation. Animal models allow for a more detailed investigation of the epigenetic profile. Pena et al. analyzed DNA methylation and histone modifications of the estrogen receptor alpha gene in the brains of dams and pups in a maternal care model [13]. These types of tissue specific, detailed epigenetic studies provide a more rigorous demonstration of epigenetic consequences.

While methylation is the best studied epigenetic mark, followed by histone modifications, the composition of our DNA is telling us that RNA may be much more important. The discovery that $<3 \%$ of the human genome encodes protein-coding genes was surprising to many people. But perhaps what is more astounding is that scientists have now found that approximately $76 \%$ of the human DNA is transcribed into some sort of RNA [14]. Much of the $76 \%$ is thought to have a regulatory role, including a role in epigenetics.

The role for noncoding RNA in epigenetics is just beginning to be understood; however, a number of studies indicate that RNA could be a powerful player in transgenerational epigenetics. The role for RNA as a mechanism of transfer of transgenerational phenotype come from experiments of microinjections of oliogribonucleotides into egg and sperm cells [15]. Given the established effect of environment on epigenetics, environmental factors such as those effecting SES could disrupt this process. While the role for noncoding RNA is still being investigated, many studies have begun to investigate how the environment can change the life course of multiple generations and the role of epigenetics in this process.

\section{Transgenerational epigenetic inheritance}

One of the most challenging and intriguing aspects of epigenetics is the transmission of modifications to subsequent generations. Transgenerational studies indicate that epigenetic modifications are not wiped clean for each generation, as was originally thought $[16,17]$. This, transgenerational epigenetic inheritance, coupled with the environmental influences of epigenetics indicates that the environment of one generation can modify the phenotype of the following generations. This farreaching epigenetic effect indicates the importance of understanding environmental influences, such as SES, on epigenetics.

The life course approach determines how the social and environmental context of one generation can affect another. For example, a maternal grandmother's exposure to neighborhood poverty during pregnancy predicts the risk for infant low birth weight and subsequent mortality among African-American women, independent of the mother's status [18]. A detailed understanding of transgenerational epigenetics is necessary to understand this complex inheritance.

\section{"The role for noncoding RNA in epigenetics is just beginning to be understood; however, a number of studies indicate that RNA could be a powerful player in transgenerational epigenetics."}

Stress is a core component of socioeconomic disadvantage. Both prenatal as well as postnatal stress has been shown to effect long-term health. Epigenetic changes in offspring exposed to maternal stress have been noted in multiple species. In Drosophila, heat stress induces phosphorylation of the transcription factor dATF-2 [19]. This leads to a loss of heterochromatin structures at several region of the genome, which is transmitted to the next generation. In mice, the early life stress of chronic unpredictable maternal separation leads to changes in behavior and alters the methylation profile in the promoter of candidate genes in the germline and brain of affected males, as well as in the germline of the offspring [20]. The licking and grooming behavior of rats has also been shown to be associated with epigenetic changes and inherited [13].

Several additional aspects of SES have been modeled in studies of transgenerational epigenetic inheritance; 
one such aspect is prenatal diet. Areas of socioeconomic disadvantage are often part of food deserts, or areas with limited access to healthy affordable food [21]. This can lead to high-fat, low-nutrient diets and contribute to SES health disparity. This type of diet has negative consequences for pregnant women, as well as subsequent generations. Animal studies have shown that maternal exposure to high-fat diet results in increased body size and reduced sensitivity to insulin through two generations via both the maternal and paternal lineages [22]. This effect continues into the third generation via the paternal lineage. This transgenerational epigenetic inheritance of diet is associated with imprinted gene patterns and changes in methylation.

Transgenerational epigenetic studies have also begun to investigate the social and racial disparity in toxicant burden. Economically disadvantaged areas have an increased risk of environmental toxin exposure [23-25]. These toxins include endocrine disruptors, heavy metals, tobacco smoke and air pollution, among others. Similar to diet studies, exposure of gestating females to environmental toxins leads to increased disease for multiple generations [3]. Also associated with the toxin derived phenotype are changes in epigenetics. Several studies have found changes in differential methylation regions, which are proposed to act as biomarkers of exposure [3].

\section{References}

1 Williams DR, Mohammed SA, Leavell J, Collins C. Race, socioeconomic status, and health: complexities, ongoing challenges, and research opportunities. Ann. NY Acad. Sci. 1186, 69-101 (2010).

2 Collins JW Jr, Soskolne GR, Rankin KM, Bennett AC. Differing first year mortality rates of term births to white, African-American, and Mexican-American US-born and foreign-born mothers. Matern. Child Health J. 17(10), 1776-1783 (2012).

3 Manikkam M, Tracey R, Guerrero-Bosagna C, Skinner MK. Plastics derived endocrine disruptors (BPA, DEHP and DBP) induce epigenetic transgenerational inheritance of obesity, reproductive disease and sperm epimutations. PLoS ONE 8(1), e55387 (2013).

4 Zhang FF, Cardarelli R, Carroll J et al. Significant differences in global genomic DNA methylation by gender and race/ethnicity in peripheral blood. Epigenetics 6(5), 623-629 (2011).

5 Tehranifar P, Wu HC, Fan X et al. Early life socioeconomic factors and genomic DNA methylation in mid-life. Epigenetics 8(1), 23-27 (2013).

6 Wu HC, Wang Q, Delgado-Cruzata L, Santella RM, Terry MB. Genomic methylation changes over time in peripheral blood mononuclear cell DNA: differences by assay type and baseline values. Cancer Epidemiol. Biomarkers Prevent. 21(8), 1314-1318 (2012).
It is clear that numerous environmental factors can contribute to an individual's epigenetic inheritance of health. The next step forward in advancing our understanding should be to integrate multiple environmental influences. This will not only create a better life course model, but the increased complexity will help to discern the causality of health disparities.

Epigenetics exists at the intersection between genetics and the environment. The goal is to use epigenetics to anticipate health in the individual and, more importantly, the population. Before this can be done, several challenges must be faced. These include investigating the nonprotein coding region of the genome, studying common diseases where the environment likely contributes to the gradation of severity, and developing new, complex models that include multiple environmental influences and have transgenerational impacts.

\section{Financial \& competing interests disclosure}

The authors have no relevant affiliations or financial involvement with any organization or entity with a financial interest in or financial conflict with the subject matter or materials discussed in the manuscript. This includes employment, consultancies, honoraria, stock ownership or options, expert testimony, grants or patents received or pending, or royalties.

No writing assistance was utilized in the production of this manuscript.

7 Adalsteinsson BT, Gudnason H, Aspelund T et al. Heterogeneity in white blood cells has potential to confound DNA methylation measurements. PloS ONE 7(10), e 46705 (2012).

8 Orkin SH,Nathan DG, Ginsburg D, Look AT, Fisher DE, Lux SE, Nathan and Oski's Hematology of Infancy and Childhood. Saunders Elsevier, PA, USA (2009).

9 Lopez MC, Palmer BE, Lawrence DA. Phenotypic differences between cord blood and adult peripheral blood. Cytometry B Clin. Cytom. 76(1), 37-46 (2009).

10 Landmann RM, Muller FB, Perini C, Wesp M, Erne P, Buhler FR. Changes of immunoregulatory cells induced by psychological and physical stress: relationship to plasma catecholamines. Clin. Exp. Immunol. 58(1), 127-135 (1984).

11 Williams DM, Johnson NW. Alterations in peripheral blood leucocyte distribution in response to local inflammatory stimuli in the rat. J. Pathol. 118(3), 129-141 (1976).

12 Bonneau RH, Sheridan JF, Feng NG, Glaser R. Stressinduced effects on cell-mediated innate and adaptive memory components of the murine immune response to herpes simplex virus infection. Brain Behav. Immun. 5(3), 274-295 (1991).

13 Pena CJ, Neugut YD, Champagne FA. Developmental timing of the effects of maternal care on gene expression and epigenetic regulation of hormone receptor levels in female rats. Endocrinology 154(11), 4340-4351 (2013).

14 Consortium TEP. An integrated encyclopedia of DNA elements in the human genome. Nature 489 (7414), 57-74 (2012). 
15 Kiani J, Grandjean V, Liebers R et al. RNA-mediated epigenetic heredity requires the cytosine methyltransferase Dnmt2. PLoS Genet. 9(5), e1003498 (2013).

16 Lane N, Dean W, Erhardt S et al. Resistance of IAPs to methylation reprogramming may provide a mechanism for epigenetic inheritance in the mouse. Genesis 35(2), 88-93 (2003).

17 Guerrero-Bosagna C, Skinner MK. Environmentally induced epigenetic transgenerational inheritance of phenotype and disease. Mol. Cell. Endocrinol. 354(1-2), 3-8 (2012).

18 Collins JW Jr, David RJ, Rankin KM, Desireddi JR. Transgenerational effect of neighborhood poverty on low birth weight among African Americans in Cook County, Illinois. Am. J. Epidemiol. 169(6), 712-717 (2009).

19 Seong K-H, Li D, Shimizu H, Nakamura R, Ishii S. Inheritance of stress-induced, ATF-2-dependent epigenetic change. Cell 145(7), 1049-1061 (2011).

20 Franklin TB, Russig H, Weiss IC et al. Epigenetic transmission of the impact of early stress across generations. Biol. Psychiatry 68(5), 408-415 (2010).
21 Beaulac J, Kristjansson E, Cummins S. A systematic review of food deserts, 1966-2007. Prevent. Chronic Dis. 6(3), A105 (2009).

22 Dunn GA, Bale TL. Maternal high-fat diet effects on third-generation female body size via the paternal lineage. Endocrinology 152(6), 2228-2236 (2011).

23 Hajat A, Diez-Roux AV, Adar SD et al. Air pollution and individual and neighborhood socioeconomic status: evidence from the multi-ethnic study of atherosclerosis (MESA). Environ. Health Perspect. 121(11-12), 1325-1333 (2013)

24 Tyrrell J, Melzer D, Henley W, Galloway TS, Osborne NJ. Associations between socioeconomic status and environmental toxicant concentrations in adults in the USA: NHANES 2001-2010. Environ. Int. 59, 328-335 (2013).

25 Vrijheid M, Martinez D, Aguilera I et al. Socioeconomic status and exposure to multiple environmental pollutants during pregnancy: evidence for environmental inequity? $J$. Epidemiol. Commun. Health 66(2), 106-113 (2012). 